\title{
Contourlet Transform for Iris Image Segmentation
}

\author{
Behrooz Zali-Vargahan \\ Department of Electrical \\ Engineering \\ Urmia University, Urmia, Iran
}

\author{
Mehdi Chehel Amirani \\ Department of Electrical \\ Engineering \\ Urmia University, Urmia, Iran
}

\author{
Hadi Seyedarabi \\ Faculty of Electrical and \\ Computer Engineering \\ Tabriz University, Tabriz, Iran
}

\begin{abstract}
The aim of this paper is improving the iris segmentation with the Contourlet transform. At first iris segmentation performed by canny edge detector and Hough Transform. By this approach some images don't segmented properly, so we want to find a way to correct the image segmentation failures. Before applying edge detector, Contourlet transform applied for image denoising. By this approach, $\% 100$ accuracy rate in iris image segmentation is obtained. Denoised image with Contourlet transform a little blurred. After image denoised and image segmented, for keep basic quality of the image, corresponded basic image and the segmented image. So, segmentation will be right on the main image.
\end{abstract}

\section{Keywords}

Canny edge detector, Contourlet transform, Hough transform, iris recognition, iris segmentation

\section{INTRODUCTION}

One of the main branches and fields of identification is iris recognition. The iris is an annular region between the black pupil and the white sclera as shown in Fig. 1 [1]. The main stages of iris recognition that have the most impact on the process can be pointed as iris segmentation, iris normalization, feature encoding and feature matching. The first preprocessing for iris recognition is iris segmentation. If iris segmentation done properly, we can hope that process works properly. But, if the first part of our process is not done correctly, we should not have expected good data for system. Thus, obvious the importance more than of the iris segmentation in iris recognition. At the image level, iris segmentation is arguably one of the most important factors driving recognition performance [2]. Two main problems occur in the iris image segmentation. The first problem is to find the boundary between the iris and the black area (pupil). The second problem is finding the boundary between iris and sclera.

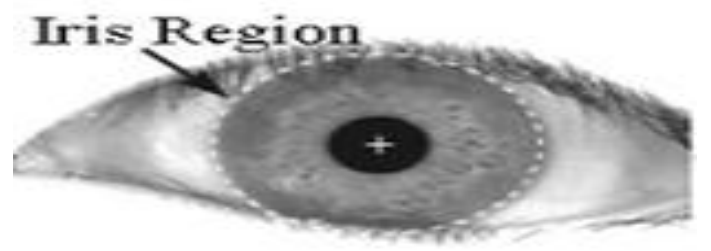

Common, the second problem is more generally. Several iris recognition algorithms have been proposed in the literature. Daugman [4-5] used a texture-based method to encode iris. Multi scale 2D Gabor Wavelet transform has used to generate a 256-byte iris code. Hamming distance is then used as a measurement to determine the proximity of two iris codes. Theintegro differential operator, which acts as a circular edge detector, is employed for determining the inner and outer boundaries of the iris as well as the upper and lower eyelids. Wildes [6] has usedLaplacian of a Gaussian (LOG) filter to extract features from the iris image. Also, a Hough transformbased method has used to segment the iris. Also, the upper and lower of the eyelidsare approximated using parabolic curves. Matching has done by using the normalized correlation between the testing and training images. Masek and Kovesi [7] employed weighted gradients using a combination of Kovesi's modified canny edge detector and the circular Hough transform to segment the iris. Huang et al. [15] first coarsely segment the iris using edge detection filters and Hough transform before normalizing it. The noise due to eyelids is then localized by the edge information based on the phase congruency. Abhyankar and Schuckers [16] use training based Active Shape Models to segment the iris from the sclera. The nonlinear shape of the iris is learned using afew training images. In this paper, iris segmentation rate improved with Contourlet transform. The output of Contourlet denoising is image that has a little blurred. This advantage increases iris segmentation rate up \%100. In this paper, CASIA database [3] is used to evaluate the performance of the proposed algorithm. In section a brief introduction about Contourlet transform presented. Section 3 includes iris segmentation with Hough transform, canny edge detector and Contourlet transform. Section 4 presents experimental results. Finally, section 5 concludes this paper.

\section{CONTOURLET TRANSFORM}

In this paper, Contourlet transform is applied for image denoising. Contourlet is an inseparable unidirectional twodimensional transform that is used to describe curves and delicate details in the images. Contourlet expansions consist of a few basic functions which tend to have different shapes and scales (non-isotropic) in different directions. Having a set of adjustable basic functions, Contourlet transform efficiently represents those flat contours that are the main components of every normal image.

Fig1: Iris image. 
Table 1. Some examples from iris segmentation and iris recognition with method

\begin{tabular}{|c|c|c|c|}
\hline Method & Segmentation Technique & Encoding Technique & Matching Technique \\
\hline Daugman [4] & Integro-differential operator & $\begin{array}{l}\text { Multiscale 2-D Gabor Wavelet } \\
\text { coefficients }\end{array}$ & Hamming distance \\
\hline Wildes [6] & $\begin{array}{l}\text { Edge detection and Hough } \\
\text { transform }\end{array}$ & $\begin{array}{l}\text { Laplacian-of-a-Gaussian (LOG) } \\
\text { filter }\end{array}$ & Normalized correlation \\
\hline Bole and Boashash [18] & Edge and contour detection & $\begin{array}{l}\text { Zero crossing of Wavelet } \\
\text { transform }\end{array}$ & $\begin{array}{l}\text { Dissimilarity function on } \\
\text { the iris }\end{array}$ \\
\hline Masek and Kovesi [7] & $\begin{array}{l}\text { Edge detection and Hough } \\
\text { transform }\end{array}$ & 1-D Log-Gabor filter & Hamming distance \\
\hline Ma et al. [8] & $\begin{array}{l}\text { Edge detection and Hough } \\
\text { transform }\end{array}$ & Circular symmetric Gabor filter & Nearest feature line \\
\hline Lim et al [9] & $\begin{array}{l}\text { Edge detection and Hough } \\
\text { transform }\end{array}$ & 2-D Haar Wavelet transform & $\begin{array}{l}\text { Competitive learning } \\
\text { neural network }\end{array}$ \\
\hline Noh at al. [19] & No information about segmentation & ICA & Hamming distance \\
\hline Ma et al. [10] & $\begin{array}{l}\text { Edge detection and Hough } \\
\text { transform }\end{array}$ & $\begin{array}{l}\text { Key local variations using } \\
\text { Wavelet transform }\end{array}$ & Exclusive OP operation \\
\hline Huang et al. [11] & $\begin{array}{l}\text { Edge detection and Hough } \\
\text { transform }\end{array}$ & Bank of Log-Gabor filter & Euclidean distance \\
\hline Huang et al. [15] & $\begin{array}{l}\text { Phase congruency and Hough } \\
\text { transform }\end{array}$ & $\begin{array}{l}\text { Multiscale 2-D Gabor Wavelet } \\
\text { coefficients }\end{array}$ & Hamming distance \\
\hline Yuan et al. [12] & $\begin{array}{l}\text { Edge detection and Hough } \\
\text { transform }\end{array}$ & 2-D phase congruency & Euclidean distance \\
\hline Dorairaj et al. [20] & Integro-differential operator & PCA and ICA & Euclidean distance \\
\hline Thornton et al. [14] & Hough transform & Advanced correlation filter & Advanced correlation filter \\
\hline He et al. [21],[22] & Pulling and pushing elastic model & Regional ordinal measure & Hamming distance \\
\hline $\begin{array}{l}\text { Abhyankar and } \\
\text { Schuckers [16] }\end{array}$ & Active Shape Models & Bi-orthogonal Wavelet & Hamming distance \\
\hline
\end{tabular}

In contrast to other transforms where the convergence precedes the discreteness, Contourlet transform starts from a discrete domain with the help of filter banks and then converges to a continuous domain, through a multi-resolution analytical framework. Contourlet transform composes two main parts: Laplacian pyramid (LP) and Directional filter banks (DFB). The original image is converted into 2 images by LP; Low-pass image and band-pass image. In the next step, each band-pass image is analyzed by DFB. Multi directional and multi scale analysis of the image is obtained by repeating the aforementioned steps on the low pass image [13]

\section{IRIS IMAGE SEGMENTATION}

As presented in the introduction, Hough transform and canny edge detector are methods that used in many papers for iris segmentation. In this paper, in addition to Hough transform and canny edge detector, Contourlet transform used for iris segmentation. In the below brief explanations about the Hough transform and canny edge detector are presented. Canny edge detector is composed of three stages. Noise attenuation in the first step. Noise can damage the image edge. The two dimensional image convolves with Gaussian window to noise reduction. The second step is to find out where it can be called the edge that used the Gradient image. Each region has a higher gradient is chosen as the edge. The third and final step is to remove points that are a little likely to be the edge. This transform can be extracted the regular shape from the image. The general trend is to the beginning, before applying edge detector; Contourlet transform applied for image denoising. Then,canny edge detector applied to find the edge of the image. After edge detector, with apply Hough transform find regular shapes that are internal and external circular iris. Using Contourlet transform for image denoising causes blurred image. Figure 2 presents some pictures that aredenoised with Contourlet transform. If you will carefully see that denoised image compared with original image have a better separation in the border area between the iris and sclera.

\section{EXPERIMENTAL RESULTS}

In this paper CASIA database [3] used. This database designed to test the identity of the iris recognition software. In this database infrared waves used to clarify the pictures. These waves cause the removal of the reflected from the eye image. Thus we don't require the calculation of removing iris area that includes reflections.At first segmentation performed without Contourlet transform. Results show about $\% 10$ of the images will fail and iris segmentation is not well. In Fig. 3 some sample pictures are shown that Hough transforms and canny edge detector alone cannot have a good segmentation. We propose to use Contourlet transform for image denoising. Denoisingapplied for image in different conditions. At first, denoising appliedto image that doesn't have any noise.Hereafter, denoising applied from image that Gaussian noise added to image with 10, 20, 30, 40, 50 variances. Fig. 4 presents some noisy images.After denoising with Contourlet transform, denoised image put to canny edge detector and Hough transform. Results show that all images in the previous section (that segmentation with Hough transform and canny 
edge detector) that have problem in segmentation, in this section segmented correctly.

In Fig. 5 some images are shown that iris segmentation aren't correct (in Fig. 3) and also see after apply our method segmentation is correct.

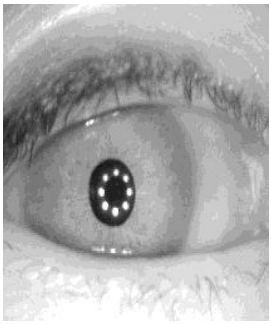

(a)
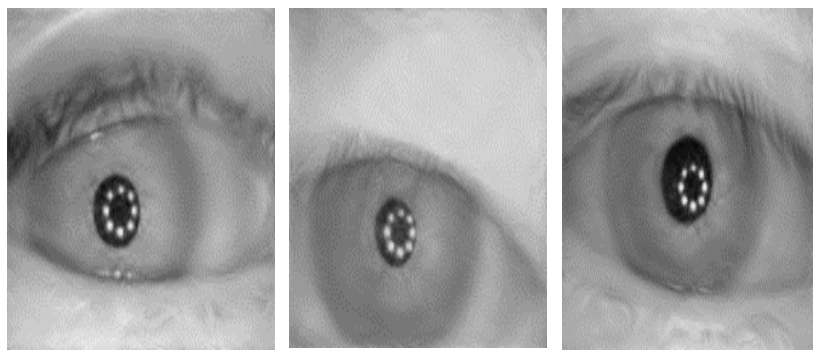

(b) Image with contourlet transform denoising

Fig 2. Denoising with Contourlet
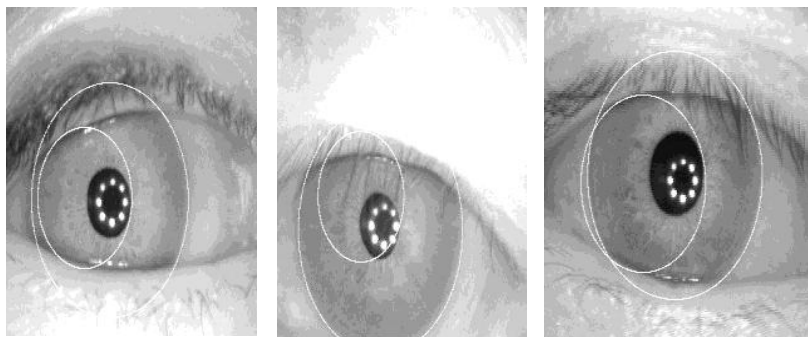

Fig 3. Non-correct segmentation

The image that denoised with Contourlet transform is little blurred and loses some quality. For image that more noise added, this problem is serious. The purpose in this paper is to find internal and external boundaries of the iris. On the other hand, we must have basic quality images for feature extraction. For this purpose after segmented denoised image, will match the original image and segmentation image then good segmentation is obtained on the original image. The best rate for our method obtained \%100.In Table 2 results of iris segmentation presented when variable noises used. Also, in this paper we compare the results of proposed method with the results of segmentation when Contourlet transform don't used. As seen Contourlet transform achieves higher segmentation rate.

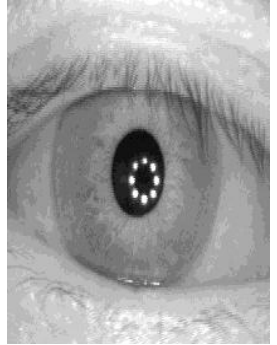

(a)

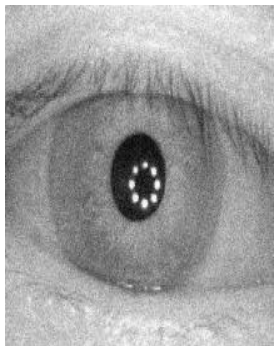

(d)

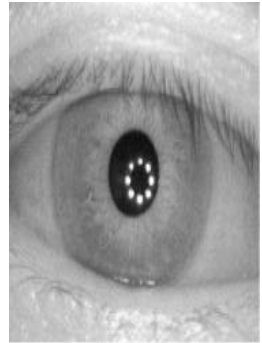

(b)

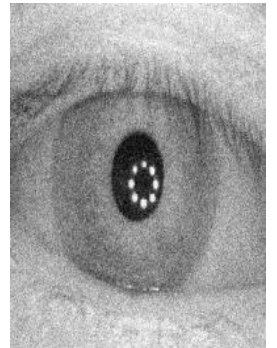

(e)

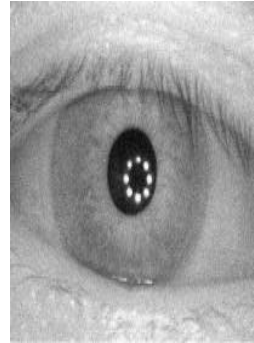

(c)

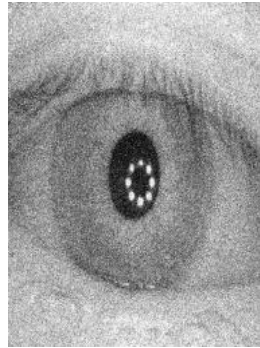

(f)
Fig 4. (A) Original image. (b)-(f) Noisy image with variance equal to $10,20,30,40$, and 50 , respectively
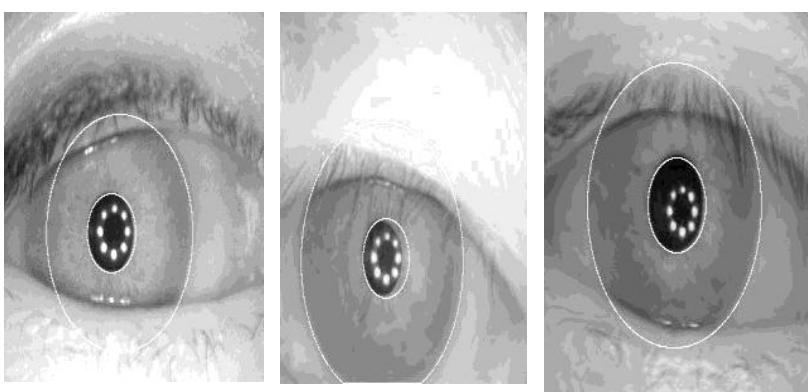

Fig 5. Correct segmentation with proposed method

Table 2.Results of segmentation rate versus noise variance

\begin{tabular}{|c||c|c|}
\hline \multicolumn{1}{|c||}{\multirow{2}{*}{$\begin{array}{c}\text { Noise } \\
\text { Variance }\end{array}$}} & \multicolumn{2}{c|}{ Segmentation Rate (\%) } \\
\cline { 2 - 3 } & $\begin{array}{c}\text { With } \\
\text { Contourlet } \\
\text { transform }\end{array}$ & $\begin{array}{c}\text { Without } \\
\text { Contourlettransform }\end{array}$ \\
\hline 0 & 95 & 94 \\
\hline 10 & 96 & 92 \\
\hline 20 & 100 & 94 \\
\hline 30 & 97 & 93 \\
\hline 40 & 96.5 & 93 \\
\hline 50 & 95.5 & 92.5 \\
\hline
\end{tabular}




\section{CONCLUSION}

One of the steps in iris recognition is iris segmentation. Good segmentation increase performance identity system. Hough transform and canny edge detector are two tools that in some papers used for iris segmentation. In this paper with adding Contourlet transform to the last two tools increase segmentation rate to \%100. In this paper, at first iris image is denoised with Contourlet transform. Image denoising results a little blurred image. Denoised image is input image for canny edge detector and then Hough transform. Results shows segmentation of the iris image with our method is extremely high efficiency. Also, we maintain basic quality of the image with corresponded basic image and the segmented image.

\section{REFERENCES}

[1] Ma, L., Tan, T., Wang, Y., Zhang, D.: 'Personal identification based on iris texture analysis, IEEE Trans. Pattern Anal. Mach. Intell., 2003, 25,(12), pp. 1519 1533

[2] J. Zuo, N.D. Kalka, and N.A. Schmid, "A robust iris segmentation procedure for unconstrained subject presentation," Biometric Consortium Conference, 2006 Biometrics Symposium: Special Session on Research, pp. 1-6, Sept. 19 2006-Aug. 212006.

[3] CASIA Iris Image Database, http://www.cbsr.ia.ac.cn/irisdatabase.htm,2009.

[4] J. G. Daugman, "High confidence visual recognition of persons by a test of statistical independence," IEEE Trans. Pattern Anal. Mach. Intell., vol. 15, no. 11, pp. 1148-1160, Nov. 1993.

[5] J. G. Daugman, "Demodulation by complex-valued wavelets for stochastic pattern recognition," Int. J. Wavelets, MultiResol. Inf. Process.,vol. 1, no. 1, pp. 117, Jan. 2003.

[6] R. P. Wildes, "Iris recognition: An emerging biometric technology,"Proc. IEEE, vol. 85, no. 9, pp. 1348-1363, Sep. 1997.

[7] L. Masek, "Recognition of human iris patterns for biometric identification," B.S. dissertation, The School of Computer Science and Software Engineering, The University of Western Australia, Crawley WA, Perth,Australia, 2003.

[8] L. Ma, Y. Wang, and T. Tan, "Iris recognition using circular symmetric filters," in Proc. 16th Int. Conf. Pattern Recogn. (ICPR), Quebec City,Canada, Aug. 2002, vol. 2, pp. 805-808.
[9] S. Lim, K. Lee, O. Byeon, and T. Kim, "Efficient iris recognition through improvement of feature vector and classifier," J. Electron.Telecommun. Res. Inst., vol. 33, no. 2, pp. 61-70, Jun. 2001.

[10] L. Ma, T. Tan, and Y. Wang, "Efficient iris recognition by characterizing key local variations," IEEE Trans. Image Process. , vol. 13, no. 6,pp. 739-750, Jun. 2004.

[11] J. Huang, L. Ma, Y. Wang, and T. Tan, "Iris model based on local orientation description," in Proc. Asian Conf. Comput.Vision , Korea,Apr. 2004, pp. 954-959.

[12] X. Yuan and P. Shi, "Iris feature extraction using 2-D phase congruency," in Proc. Third Int. Conf. Inf. Technol. Appl. (ICITA), Sydney,Australia, Jul. 2005, vol. 33, pp. 437-441.

[13] Do, M. N., \& Vetterli, M. (2003). Contourlets beyond wavelets. New York: J. Stoeckler and G.V. Welland, Eds. Academic Press.

[14] J. Thornton, M. Savvides, and B. V. Kumar, "Robust iris recognition using advanced correlation techniques," in Proc.Second Int. Conf. Image Anal.Recogn. (ICIAR), Toronto, Canada, Sep. 2005, vol. 3656,pp. 1098-1105, Springer Berlin/Heidelberg.

[15] J. Huang, Y. Wang, T. Tan, and J. Cui, "A new iris segmentation method for recognition," in Proc. 17th Int Conf. Pattern Recogn.(ICPR), Cambridge, U.K., Aug. 2004, vol. 3, pp. 23-26.

[16] A. Abhyankar and S. Schuckers, "Active shape models for effective iris segmentation," in Proc. SPIE Conf. Biometric Technol. Human Identif.III, Orlando, FL, Apr. 2006, pp. 62020H.1-62020H.10.

[18] W. Boles and B. Boashash, "A human identification technique using images of the iris and wavelet transform," IEEE Trans. Signal Process. ,vol. 46, no. 4, pp. 1185-1188, Apr. 1998.

[19] K. Bae, S. Noh, and J. Kim, "Iris feature extraction using independent component analysis," in Proc. 4th Int. Conf. Audio and VideoBased Biometric Person Authentic. (AVBPA), Guildford, U.K., 2003,pp. 838-844.

[20] V. Dorairaj, N. A. Schmid, and G. Fahmy, "Performance evaluation of iris based recognition system implementing PCA and ICA encoding techniques," in Proc. SPIE Conf. Biometric Technol. Human Identif.III, Orlando, FL, Apr. 2005.

[21] Z. He, Z. Sun, T. Tan, X. Qiu, C. Zhong, and W. Dong, "Boosting ordinal features for accurate and fast iris recognition," in Proc. IEEE Comput. Soc. Workshop Biometrics at the Computer Vision Pattern Recogn.Conf., 2008. 